\title{
Uncertainty Propagation in an Integrated Land Use-Transportation Modeling Framework: Output Variation via UrbanSim
}

\author{
Anant Pradhan \\ Graduate Student Researcher \\ The University of Texas at Austin. \\ 6.9 E. Cockrell Jr. Hall \\ Austin, TX 78712-1076. \\ Email: anant.pradhan@ mail.utexas.edu, \\ Phone: 512-232-6599 \\ and \\ Kara Maria Kockelman (Corresponding Author) Clare Boothe Luce Professor of Civil \\ Engineering The University of Texas at Austin \\ 6.9 E. Cockrell Jr. Hall \\ Austin, TX 78712-1076 \\ Email: kkockelm@mail.utexas.edu \\ Phone: 512-471-0210, \\ FAX: 512-475-8744 \\ The following paper is a pre-print and the final publication can be found in \\ Transportation Research Record No. 1805: 128-135, 2002. \\ Presented at the 81st Annual Meeting of the Transportation Research Board, \\ January 2002
}

\begin{abstract}
This study examines the impact of uncertainty in the land use component of a partially integrated land use-transportation modeling system called UrbanSim. Outputs from the land use model (LUM) act as inputs for a traditional 4-step travel demand model (TDM), and travel times from the traffic-assignment stage of the TDM are fed forward into the subsequent years LUM. This work examines the propagation of uncertainty across model stages as well as at each model stage over time. A factorized design approach is used to model uncertainty in demographic inputs (which include aggregate growth rates and mobility rates) to the land use model, as well as uncertainty in various model parameters. The results suggest that while several model inputs may affect model outputs in the short run, only those inputs that have a cumulative effect are likely to have a significant impact on outputs in the long run. The results also suggest that uncertainty in model outputs may increase for the first few years for which the model is run, as modified inputs send shocks through the urban system. However, the level of uncertainty appears to come down in later years, as households, jobs, and developers respond to changed input conditions
\end{abstract}

\section{Keywords}

Error propagation, uncertainty representation, integrated models, land use, travel demand model 


\section{INTRODUCTION}

Previous studies have shown that transportation planners often do not recognize the uncertainty implicit in modeling a complex and dynamic urban system (please see Mehndiratta et al [2000] for a discussion of how issues related to uncertainty are presently addressed in metropolitan planning organizations). Typically, static models are used to predict discrete values of travel demand, mode shares, and traffic volumes on links (Niles and Nelson, 2001). Uncertainty present in earlier model stages also tends to compound itself over later stages, when model stages are developed sequentially (Zhao and Kockelman, 2001).

The Intermodal Surface Transportation Efficiency Act (ISTEA) of 1991 requires that metropolitan and statewide transportation plans be integrated with land use plans (Miller et al, 1999). However, few studies in the past have examined uncertainty propagation in the context of an integrated land use-transportation modeling framework.

The objective of this study is a better understanding of uncertainty in demographic and other inputs on urban development, how this uncertainty is propagated through the stages of an in integrated land use-transportation model, and how uncertainty evolves over time. This has been accomplished by outlining a general method of uncertainty analysis that can be applied to a variety of land use-transportation models. The results of applying this model to a land usetransportation modeling system called UrbanSim have been presented, along with certain conclusions, some of which are generic to many such models.

\section{BACKGROUND}

This section reviews literature on the sources of uncertainty in land use-transportation models, the popular techniques employed for representing uncertainty, and other studies that have examined uncertainty and its propagation.

\section{Sources of Uncertainty}

Of the many possible sources of error in travel and emission models, the most important ones are thought to be natural uncertainty, uncertainty in model design and structure, data uncertainty and calibration errors, and uncertainty in socioeconomic projections and other model inputs.

Natural uncertainty includes the uncertainty due to quantities that are random even in principle, and quantities that are random because of the effort and cost involved in precise measurement (Frey, 1992). The structure of the mathematical model used to represent urban behavior is also a key source of uncertainty, because models are necessarily simplified representations of the phenomena being studied. In addition, models valid in one portion of the input space may be completely inappropriate for making predictions in other regions of the input space, or in the same portion at a different point in time ${ }^{1}$ (Niles and Nelson, 2000; Frey, 1992). Data uncertainty and calibration errors include measurement errors due to imprecision, systematic biases, estimation of parameters using small samples, and estimation of parameters using non- 
representative samples (Frey, 1992; Stopher and Meyburg, 1975). Harvey and Deakin (1995) suggest that uncertainty in socioeconomic projections and other inputs are possibly the greatest source of uncertainty in any model. They are especially significant since modelers can do very little to reduce the errors due to these sources (Barton-Aschman, 1997).

The relative importance of error sources (Pell, 1984) can be judged based on the sensitivity of forecasts to input errors and the magnitude of forecast errors. These relationships are capably measured by elasticities and coefficients of variation respectively.

\section{Approaches to Uncertainty Representation}

The most popular ways of representing uncertainty are interval mathematics (Moore, 1996), fuzzy theory (Klir and Yuan, 1995), and probabilistic analysis (Papoulis, 1991). The objective of interval mathematics is to estimate upper and lower limits on model predictions due to parameters that are known to be unknown but bounded. Fuzzy theory can be used to represent uncertainty through the process of fuzzification ${ }^{2}$. In the probabilistic approach, uncertainties associated with model inputs are described by probability distributions, the objective being estimation of the output probability distribution. Probabilistic methods include analytical methods and sampling based methods.

Sampling based methods involve running the original model for a set of input and parameter combinations, and using the model outputs at those points to estimate model sensitivity. Some of the widely used sampling based uncertainty analysis methods are the Monte Carlo and Latin Hypercube methods (Fishman, 1996), Fourier Amplitude Sensitivity Test (FAST) methods (McRae et al, 1996), reliability based methods (Bjerager, 1990), and response surface methods (Fedorov, 1983). Of these, Monte Carlo methods are judged the most suitable for analyzing uncertainty in complex urban systems.

Monte Carlo (MC) methods involve random sampling from the distribution of inputs, and successive model runs until a statistically significant distribution of outputs is obtained. These can be used to solve problems with probability structures, or non-probabilistic problems such as finding the area under a curve. However, these methods require a large number of samples. In order to achieve computational efficiency, methods that sample from the input distribution in an efficient manner have been introduced. One such variant of the standard Monte Carlo method is the Latin Hypercube (LH) sampling method. In this method, the range of probable values for each input parameter is divided into ordered segments such that the parameter space, consisting of all uncertain parameters, is partitioned into cells having an equal probability. Thus, parameter estimates are sampled in an efficient manner, since each parameter is sampled only once from each of its possible segments. The advantage of this approach is that it allows representation of the extremes of the probability distribution of the outputs.

The current study employs a factorized design approach, which is similar to the LH method in that it also involves dividing the input parameter space is divided into equal probability bins. However, rather than randomly sample points from within the bin, the mid-percentile point of each bin is selected as the sample point. As explained in the 'Uncertainty in the Land Use 
Component' section, it represents an efficient way of obtaining a well-distributed range of input values.

\section{Previous Studies}

Zhao and Kockelman (2001) conducted a study of the propagation of uncertainty in 4-step travel demand models. They used Monte Carlo simulation and sensitivity analysis to quantify variability in model outputs. Their results suggested that uncertainty is compounded over the first three stages of a transport model, resulting in a magnification of errors at later stages. However, traffic assignment, the final step of the model, reduced these variations back to the initial, input levels. The authors hypothesized that this was due to a centralizing tendency of compounded travel choices, and congestion feedbacks in the shortest-path search algorithm.

Harvey and Deakin (1995) conducted a similar study, in which they considered uncertainty in population growth, fuel price and household income levels in the STEP models of the Los Angeles (LA) region. They found that plausible ranges of the input variables resulted in percentage changes in total VMT of $25 \%$ below to $15 \%$ above the original prediction.

A study conducted by Thompson et al (1997) examined the impact of higher-than-projected population estimates on emission trends for metropolitan regions in California. They found that levels of $\mathrm{CO}, \mathrm{ROG}$ and $\mathrm{NO}_{\mathrm{x}}$ were below projected attainment year levels, even for high growth scenarios, as a result of fuel and motor vehicle emissions control programs.

Rodier and Johnston (2001) identified plausible error ranges of $\pm 2 \%$ for population and employment growth projections. Their results suggest that while plausible error ranges for household income and fuel prices may not be a significant source of uncertainty, plausible errors in population and employment projections are likely to cause significant errors in predictions.

There are a few other studies that have focused on uncertainty in travel demand modeling. The earliest studies (Robbins, 1978; Bonsall et al, 1977) attempted to identify the possible errors in each step, and stressed the importance of sensitivity analysis. Studies have also focused on simulation techniques (Ashley, 1980) by employing a multivariate probability distribution to represent correlated inputs.

The current study is based on the work of Zhao and Kockelman (2001), but differs in two important aspects. First, it investigates the uncertainty associated with the land use-component of a land use-transportation modeling system (UrbanSim). And, second, it considers the evolution of uncertainty over time, in addition to its propagation across model stages.

\section{CURRENT APPLICATION}

Of the many sources of uncertainty outlined in the previous section, not all can be dealt with empirically. This study primarily considers only those sources of uncertainty deemed amenable to empirical analysis. These include both data uncertainty, and uncertainty in socioeconomic 
inputs to the model. However, a brief discussion of the weakness of UrbanSim as a modeling system has been presented in the following system.

This work examines the impact of uncertainty in the land use component of a partially integrated $^{3}$ land use-transportation modeling system known as UrbanSim. Here, outputs from the land use model (LUM) act as inputs for a traditional 4-step travel demand model (TDM); and where travel times from the traffic-assignment stage of the TDM are fed forward into the subsequent years LUM.

Uncertainty in model outputs can be attributed to uncertainty in model inputs (for example, population and employment forecasts) and/or uncertainty in model parameters (for example, coefficients in a trip generation model). This study proposes to examine the impact of, and quantify the sensitivity to, both of these sources.

\section{Land Use Component}

This analysis was carried out for Eugene-Springfield, Oregon using 271 traffic analysis zones (TAZ's). Urban development was modeled using UrbanSim (Waddell et al, 2001; UrbanSim Reference Guide, 2000; UrbanSim Application Guide, 2000), an urban simulation model that incorporates the interactions of individual decisions taken by households, businesses, developers and governments. UrbanSim is implemented as the interaction of a set of sub-models that simulate the mobility and location choices of household and businesses, the development choices of real estate developers, and the policy constraints imposed by governments. The software has been tested in Eugene-Springfield, Oregon, and subsequently applied in Honolulu, Hawaii and Salt Lake City, Utah.

UrbanSim draws on random utility theory for the theoretical foundation of its discrete choice models, and builds on techniques of disaggregate choice modeling widely used in mode choice models, extending the discrete choice framework to households and businesses. UrbanSim incorporates a dynamic disequilibrium approach, representing adjustment processes that occur at different rates, unlike the cross-sectional equilibrium approach adopted by models such as DRAM/EMPAL (Putman, 1983), MEPLAN (Abraham and Hunt, 1999) and TRANUS (De la Barra, 1995) - which can be difficult to sustain when considering the complex interactions among urban housing, labor and transportation markets, since equilibrium may not be realistic in a complex and evolving urban system. The UrbanSim software developed as an Open Source project using the GNU General Public License, which means that anyone can freely access the source code, modify it, and redistribute it. The salient features of UrbanSim are an explicit accounting of land, structures and occupants; microsimulation at the grid cell ${ }^{4}$ level of mobility and location choices of individual households and jobs; microsimulation at the grid cell level of land development and redevelopment; and disaggregation of household and employment location choices to the level of grid cells (Waddell et al., 2001).

Model components include the economic and demographic transition models, the household and employment mobility models, the accessibility model, the household and employment location choice models, the real estate development model, and the land price model. These have been described below: 
- Economic Transition Model: The model integrates exogenous forecasts of aggregate employment into the model database by computing sectoral growth or decline, and removes jobs from declining sectors and queues jobs (for the location choice model) in sectors that experience growth.

- Demographic Transition Model: The model integrates changes in the distribution of households by type into the model database, by adding household births to a list (for the location choice model) and deleting households from the database (in case of household deaths).

- Employment and Household Mobility Models: These models predict the probability of jobs and households of each type moving from their current locations respectively.

- Employment and Household Location Choice Models: These models predict the probabilities of location of different sites for new and moving jobs and households respectively.

- Household Mobility Model: The model predicts the probability that households of each type move from their current locations.

- Land Price Model: The model uses a hedonic regression equation to predict land prices, which act as indicators of the match between demand and supply of land for various development types at different locations.

- Real Estate Development Model: The model predicts the probability that a grid cell will experience a development event in any simulation year, and it identifies the type of event that is most likely.

Some of UrbanSim's limitations are mentioned here. UrbanSim does not have a travel model component and, therefore, requires manual intervention to run an exogenous travel model and update the interzonal travel times, which in turn affect accessibility. This makes the model slow and cumbersome to work with. Due to the nature of Urbansim, the data requirements are extreme. Urbansim requires a spatial representation of the urban environment being modeled at a very disaggregate level, thereby increasing the possibility of calibration errors. The model does not account for commodity flows, which are an important component of traffic flow in urban systems. The time lags (one year in the current implementation) between land use and transportation systems have not been tested for realism. Jobs and households move one at a time (via a queue of movers) rather than interacting simultaneously. The land price model is not yet calibrated to account for excess supply in the land market - a critical determinant of land values.

The current application uses the Eugene-Springfield dataset, which was provided by the University of Washington. The Lane Council of Governments (LCOG), a voluntary association of local governments in Lane County, Oregon that facilitates multi-jurisdictional planning and program development, provided the Eugene-Springfield network, which comprises 2970 links. The outputs from the land use component were fed into a traditional 4-step travel demand model, which has been implemented in TransCAD (Caliper, 2000). The travel model was run every five years (in 1980, 1985, 1990, and 1995), as recommended by UrbanSim's creators (Waddell et al, 2001). The resulting estimates of inter-zonal travel times were fed forward into subsequent years' land use models. 


\section{UNCERTAINTY IN THE LAND USE COMPONENT}

This study emphasizes the uncertainty in UrbanSim's land use component arising from uncertain demographic inputs, and model parameters. While uncertain socioeconomic forecasts are regarded as the major sources of uncertainty and have been the focus of many of the previous studies (Rodier and Johnston, 2001; Thompson et al, 1997; Harvey and Deakin, 1995) not much attention has been paid to the fact that most models are based on parameter estimates governing relationships that are thought to exist in the population, and that these relationships may vary over time.

While there are well-established guidelines for choosing a distribution to represent the distribution of parameter estimates (for example, those obtained by ordinary least squares or maximum likelihood estimation have an asymptotically normal distribution), the choice of a distribution to represent the uncertainty in model inputs (for example, population growth rate) is less clear-cut.

Given a probability distribution to represent inputs, one may choose sample points based on MC or LH methods. In the current study, given the desire to maintain a reasonable number of model runs, the factorized design approach outlined earlier was adopted, wherein the input distribution was divided into three equal-probability bins, and the mid-percentile point of each bin was chosen as the sample point. This has been illustrated in Figure 1, and represents an efficient way of obtaining well-distributed inputs.

\section{Population and Employment Growth Rates}

In this work, annual population and employment growth rates were varied from a high of 1.5 times, to a low of 0.5 times the default growth rate. Together with the mean, these represent three well-distributed sample points. Population and employment growth rates were varied in tandem based on an expectation of high positive correlation and a desire to maintain a reasonable number of model runs (each of which requires several hours of computing time and cumbersome manual intervention).

\section{Household and Employment Mobility Rates}

Household and employment mobility rates represent the probability that an individual household or job will be allowed to join the queue of locators waiting to choose locations in a particular model year. These rates were varied from a high of $30 \%$ to a low of $10 \%$, with the average mobility rate being set to $20 \%$. The mobility rates were varied in tandem for the reasons described above.

\section{Location Choice Coefficients}

A very significant factor in the location choice of a household or a job is the accessibility of the location (represented by the log of accessibility to employment for the residential location choice 
model, and the log of accessibility to households for the employment location choice models). The coefficient on this variable was varied to equal its $17^{\text {th }}, 50^{\text {th }}$ and $83^{\text {rd }}$ percentiles ${ }^{5}$ for both the residential, and the employment location choice models.

\section{Land Price Coefficient}

Accessibility to employment (represented by the log of total employment within $600 \mathrm{~m}$ of the grid cell) is also a significant determinant of land values. The coefficient for this variable has been varied to equal its $17^{\text {th }}, 50^{\text {th }}$ and $83^{\text {rd }}$ percentiles for the land price model.

In all, 81 combinations of the variations, or 81 scenarios have been modeled.

\section{RESULTS}

The sensitivities of model outputs to input values were determined by regressing outputs on input transformation parameters. The statistical and practical significance of any independent variable can be assessed by its p-value and its standardized coefficient. A p-value is the level of significance for a two tailed t-test of the hypothesis that the variable has no impact on the dependent variable (i.e., its regression coefficient is zero). A variable with a p-value of less than 0.05 is considered very statistically significant. A standardized coefficient may be calculated as shown below:

$$
\beta_{x}^{s t d}=\beta_{x} \times \frac{\sigma_{x}}{\sigma_{y}}
$$

Where $\beta_{x}^{\text {std }}$ is the standardized coefficient, $\beta_{x}$ is the regression coefficient, $\sigma_{x}$ is the standard deviation of the dependent variable, and $\sigma_{y}$ is the standard deviation of the dependent variable.

Thus, the standardized coefficient represents the change in the dependent variable (measured in multiples of its standard deviations) caused by a change of one standard deviation in the independent variable.

The outputs of both the land use and the travel demand models were considered for analysis. Outputs of the land use model that were examined in detail here are land prices, occupancy rates and occupancy densities. Occupancy rate (Occ. Rate) is defined as the proportion of available dwelling units that are occupied by households or jobs. Occupancy density (Occ. Dens.) is defined as the proportion of available developed area that is used. Both occupancy rate and occupancy density are clearly bounded by zero and one. In order to assess the impact at a disaggregate level, land use outputs for three different zones were also tracked. These zones were a suburban zone, a rural zone, an urban residential zone, and a zone in the CBD area.

Outputs of the travel demand model that were examined include the vehicle miles traveled (VMT), the vehicle hours traveled (VHT), and the average of flows on a few individual links. It was observed that the same three links carried the three highest flows for all scenarios in all 
years. These links were not, however, the most congested in the network (the average volume to capacity ratios on these links varied 0.7 to 0.3 ). The average of the individual flows (Avg. Flow) on the highest-flow link, the second-highest flow link, and the third-highest flow link was tracked in order to assess the impact of uncertainty at a spatially disaggregate level on what are likely to be critical links on the travel network. The highest and second-highest flow links are in urban areas, whereas the third-highest flow link is in a rural area.

The results of the analysis for three years $(1985,1990$, and 1995) are summarized in Tables 1 and 2 . The acronyms used are those defined in this section. A simple example of how the values in Tables 1 and 2 may be interpreted is presented below. Consider the sensitivity of land use outputs to growth rates and mobility rates in 1985. The relatively large magnitude of the standardized coefficient and the small p-value for growth rates indicate that growth rates have an impact that is both statistically significant, and significant in practical terms. However, the relatively small magnitude of the standardized coefficient for mobility rates indicates that while their impact is statistically significant (indicated by a small p-value), it is not significant in practical terms.

The results indicate that population and employment growth rates are a significant determinant of land prices. Higher growth rates are associated with lower land values, possibly because access to employment (as measured by the log of the employment accessibility measure, which is not the same as the employment within 600m) has a negative impact on land values. Not surprisingly, the coefficient of the land price model has a very significant impact on land prices that is consistent with the sign of the coefficient. Higher mobility rates are associated with lower land values, possibly because they allow households to respond to higher land values by shifting away from high-value areas. However, this impact is significant only for the first few years of the model run. Presumably, this is because of the congestion that occurs in later years, which prevents households from relocating in low-value areas.

Higher population and employment growth rates also result in higher occupancy rates and occupancy densities. This effect however appears to diminish in later years, as builders respond to higher occupancy rates and densities. Mobility rates appear to impact occupancy rates and densities only in the short run, possibly because of the reasons mentioned above

Disaggregate outputs of the land use model, not presented in this paper, follow a similar trend, although the results may not be as significant statistically, given the greater scope for unobserved variations at such a disaggregate level. As expected, variation in growth rates is the major source of variation in model outputs, even at a disaggregate level. Mobility rates do impact some outputs of the land use model in the initial years of the model run, although the trend is not very consistent because of increased unobserved variation in model outputs at the disaggregate level.

Population and employment growth rates have a very significant positive impact on aggregate as well as disaggregate outputs of the travel demand model, for obvious reasons. Mobility rates have a statistically significant negative impact on VHT, VMT, as well as the average of the three highest flows in the first few years of the model run. This may be because higher mobility rates allow households and jobs to relocate in more accessible zones, thus reducing total travel in the 
system. However, this impact is dampened in later years of the model run, possibly because of the increased congestion in the urban system.

A less obvious finding is that a higher value of accessibility (as measured by the log of the employment accessibility for the residential location choice model, and the log of population accessibility for employment location choice models) appears to be associated with higher VHT and VMT, as well as higher flows on individual links in the short run, although this impact is not significant in practical terms. Because of the complex interactions of the location choice models, it is difficult to predict the impact that any one coefficient in these models may have on model outputs, although it is expected that this impact would not be significant in practical terms. In the long run however, accessibility does not appear to have a significant impact on any of the outputs of the travel demand model.

In the long run, only population and employment growth rates appear to have a significant impact on all model outputs. One possible explanation for this could be the fact that only population and employment growth rates have an impact that is cumulative. In the long run, the cumulative effects of differences in growth rates appear to overshadow all other differences. This is also reflected in the frequency distribution of model outputs, which are split into distinct groups on the basis of differences in growth rates.

The evolution of uncertainty over time is depicted in Figure 2. The level of uncertainty (as measured by the coefficient of variation [COV], which is defined as the standard deviation of a variable divided by its mean) appears to go up in the initial years for which the model is run, but then goes down. This is a trend that is consistently reflected in all outputs of both the land use and the travel demand model. This may be because changes in input data cause shocks throughout the urban system being modeled, and these shocks build up in the initial years for which the model is run. These shocks are caused by the fact that changes made to the input data may be in discord with existing model conditions. As households, jobs, and developers respond to the changed input conditions (via their respective models), these shocks are dampened, resulting in a lowered level of uncertainty in later years.

The evolution of uncertainty in disaggregate outputs of the land use model follow a similar trend, although the level of uncertainty itself (as measured by the COV) is much higher at the disaggregate level. Typically, the variation in occupancy rates and densities is larger for the less congested rural and suburban zones. Because the urban residential zone and the CBD zone approach saturation in later years, there is little variation in occupancy rates and densities in these zones, although land values show significant variation even in later years of the model run.

Significantly, outputs of the travel demand model appear to be far less variable than outputs of the land use model. It is hypothesized that this may be a direct consequence of the traffic assignment procedure employed, which is the Stochastic User Equilibrium (Sheffi, 1984; Sheffi and Powell, 1982) assignment. The SUE procedure attempts to find an equilibrium in traffic flows through a large number of iterations, which may have the effect of reducing the variability in link flows, and hence in total VHT and VMT. 
The current work does not explicitly incorporate the possibility of bias in model outputs. However, a comparison of model outputs in 1994 with observed patterns in Eugene-Springfield in 1994 has been presented in Table 3. Most outputs of the land use and transportation model have a very small bias, indicating that model predictions are fairly close to observed patterns in Eugene-Springfield.

A second source of uncertainty arose when replicate runs of a particular scenario were performed with differing seeds for UrbanSim's random number generator. This analysis was carried out using a more recent version of UrbanSim (version 1.0) that provided the added flexibility required. However, this analysis was carried out for a different (albeit comparable) period of 15 years (from 1995 to 2010), since the newer version of UrbanSim was configured to run for this specific period. As before, the travel demand model was run every five years (in 1995, 2000, 2005, and 2010), and the updated travel times fed forward into the subsequent years land use model. All inputs were maintained at the default level. Because of the excessive time required to perform each run, 10 replicate runs were deemed sufficient for providing a general sense of the magnitude of uncertainty involved. As expected, the uncertainty resulting from variation in random number seeds was appreciably smaller than the uncertainty resulting from uncertainty in UrbanSim's inputs, and appears to increase continuously for all the years that the model was run.

\section{LIMITATIONS}

The scope of the current work was constrained by a desire to maintain a reasonable number of model runs, given the time required to complete each run. As a result, several key parameters (for example, other variables in the location choice models and the land price model, as well as variables in the real estate development model; space utilization per employee; and household car ownership probabilities) were not varied. Since population and employment growth rates were varied in tandem, it was not possible to assess the sensitivity of model outputs to each of these individually. Because of the relatively few sample points of model inputs, the distribution of model outputs could not be examined. However, the 81 experiments allowed a factorized design that uniformly sampled the parameter space of interest, given the considerable time requirements of each simulation run.

There was also little congestion ${ }^{6}$ on the network, which suggests that these results may not be valid for situations in which there is significant congestion on the network. It is also not clear whether any of the results are affected by input variations that were not controlled for ${ }^{7}$, but may have affected the results of the study. Lastly, the validity of all results is constrained by the limitations of UrbanSim as integrated land use-transportation modeling software, and by the appropriateness of the travel demand model implemented.

\section{CONCLUSIONS}

This study confirms some of the results of previous studies (Harvey and Deakin, 1995), and suggests a few new results. For instance, uncertainty in model parameter estimates may be a significant source of uncertainty in some model outputs. However, in the long run, only those inputs that have a cumulative effect are likely to have a significant impact on model outputs. 
Although these results were derived using one particular land use-transportation modeling system, they are possibly applicable to many such models. The level of uncertainty appears to increase as input shocks build up in the urban system, but then appears to fall as urban actors respond to changes in input conditions. While it may be inappropriate to generalize this result to all land use-transportation models, it is suggested that they are generic to all applications of micro-simulation based models in congested urban systems. However, the validity of these results is constrained by the appropriateness of UrbanSim as an integrated land usetransportation modeling system.

Because land use-transportation models are usually used to forecast urban conditions several years in the future, it is important to understand how uncertainty in forecasts evolves over time. It is not clear, however, whether the results of the study are valid for all land use-transportation models in general, or whether they are a reflection of the shortcomings of UrabanSim in particular.

Future work should consider other inputs that were not varied in the current application, particularly those that may have had an unobserved effect on the results of the current study. It should also consider a realistic correlation between inputs. While a well-distributed range of inputs is important, in order to obtain a clear sense of the distribution of outputs, the scope of any such work is limited by the time required to complete each simulation run. In order to generalize the results to all land use-transportation models, uncertainty propagation in other land usetransportation models (for example, ITLUP) should also be considered. Of course, future work should also consider other sources of uncertainty, which may be due to misspecification of the model structure and the underlying random nature of behavior.

The results of this study are of great value to all planning agencies that are now moving towards integrated land use-transportation models for making forecasts of future land use and transportation patterns. While the methods employed in the current study cannot provide a measure of how far forecasts may be from actual future patterns, they do provide a sense of how much of this uncertainty can be attributed to uncertainty in model inputs.

\section{ACKNOWLEDGEMENTS}

The authors of this paper wish to thank the Southwest Region University Transportation Center for its financial support, Paul Waddell and the University of Washington for their provision of UrbanSim, Howard Slavin and the Caliper Corporation for their provision of TransCAD, and Bud Reiff and the Lane Council of Governments for their provision of data and other support.

\section{ENDNOTES}

${ }^{1}$ This source of uncertainty is also known as the " $\Delta \mathrm{E}$ caveat".

${ }^{2}$ This process is used to generalize any set from a crisp (discrete) form to a fuzzy (continuous) form.

${ }^{3}$ The model is partially integrated since the TDM is exogenous to the modeling system. In a fully integrated model, such as ITLUP, travel demand is derived directly from the LUM. 
${ }^{4}$ In the current implementation, a grid cell is sized $150 \mathrm{~m}$ by $150 \mathrm{~m}$.

${ }^{5}$ For the reasons described above.

${ }^{6}$ The average volume to capacity ratio on the network varied from 0.19 , for high growth scenarios, to 0.17 , for low growth scenarios. The maximum volume-to-capacity ratio varied from 1.67 , for high growth scenarios, to 1.57 , for low growth scenarios.

${ }^{7}$ While every attempt was made to eliminate any such input shocks, the sheer volume of input data required for running UrbanSim makes it impossible to consider the impact of every possible input. 


\section{REFERENCES}

Abraham, J. E. and J. D. Hunt. Firm Location in the MEPLAN Model of Sacramento. Transportation Research Record 1685, TRB, National Research Council, Washington, D.C., 1999, pp. 187-198.

Ashley, D. J. Uncertainty in the Context of Highway Appraisal. Transportation 9(3), 1980, pp. 249-267.

Barton-Aschman Associates Inc., and Cambridge Systematics Inc. Model Validation and Reasonableness Checking Manual. Federal Highway Administration Report, Washington, D. C., 1997.

Bonsall, P. W., A. F. Champerwone, A. C. Mason, and A. G. Wilson. Transport Modeling: Sensitivity Analysis and Policy Testing. Progress in Planning 7(3), 1977.

Ben-Akiva, M. and S. Lerman. Discrete Choice Analysis: Theory and Application to Transportation Demand. MIT Press, Cambridge, Massachusetts, 1985.

Bjerager, P. On Computation Methods for Structural Reliability Analysis. Structural Safety, Vol. 9(2), 1990, pp. 79-96.

Caliper Corporation. Travel Demand Modeling With TransCAD 4.0. Caliper Corporation, Newton, Massachusetts, 2001.

De la Barra, T. Integrated Land Use and Transportation Modeling: Decision Chains and Hierarchies. Cambridge University Press, 1995.

Fedorov, V. V. Analysis and Design of Simulation Experiments for the Approximation of Models. IIASA, WP-83-71, 1983.

Fishman, G. S. Monte Carlo: Concepts, Algorithms, and Applications. Springer-Verlag, New York, 1996.

Frey, H.C. Quantitative Analysis of Uncertainty and Variability in Environmental Policy Making. AAAS/EPA Environmental Science and Engineering Fellows Program, American Association for the Advancement of Science, Washington, DC, 1992.

Harvey, G. and Deakin, E. Description of the STEP Analysis Package, 1995, Berkeley, California.

Klir, J. G. and B. Yuan. Fuzzy Sets and Fuzzy Logic: Theory and Applications. Prentice Hall, Englewood Cliffs, New Jersey, 1995. 
McRae, G. J., J. W. Tilden, and J. H. Seinfeld. Global Sensitivity Analysis: A Computational Implementation of the Fourier Amplitude Sensitivity Test (FAST). Computers and Chemical Engineering, Vol. 19 (Suppl.): S779-S784, 1995.

Mehndiratta, S. R., D. Brand, and T. E. Parody. How Transportation Planners and Decision Makers Address Risk and Uncertainty. In Transportation Research Record 1706, TRB, National Research Council, Washington, D.C., 2000, pp. 46-53.

Miller, E. J., D. S Kriger, and J. D. Hunt. Research and Development Program for Integrated Urban Models. Transportation Research Record 1685, TRB, National Research Council, Washington D. C., 1999.

Moore, R. E. Interval Analysis. Prentice Hall, Englewood Cliffs, New Jersey, 1966.

NCHRP. Travel Estimation Techniques for Urban Planning. NCHRP report 365, TRB, National Research Council, 1995.

NCHRP. Quick-Response Urban Travel Estimation Techniques and Transferable Parameters. NCHRP report 178, TRB, National Research Council, 1978.

Niles, J. S. and D. Nelson. Identifying Uncertainties in Forecasts of Travel Demand. Preprint for the Transportation Research Board's 80 ${ }^{\text {th }}$ Annual Meeting, Washington D.C., Jan 2001.

Papoulis, A. Probability, Random Variables, and Stochastic Processes. McGraw-Hill, New York, 1991.

Parsons Brinckerhoff Quade and Douglas. Nested Logit Mode Choice Model Specifications. Report prepared for the Lane Council of Governments, November 1995.

Pell, C. M. The Analysis of Uncertainty in Urban Transportation Planning Forecasts. PhD Dissertation, Cornell University, 1984.

Putman, S. Integrated Urban Models: Policy Analysis of Transportation and Land Use. Pion, London, 1983.

Robbins, J. Mathematical Modeling - the Errors of Our Ways. Traffic Engineering and Control, January 1978, pp. 32-35.

Rodier, C. J. and R. A. Johnston. Uncertain Socioeconomic Projections Used in Travel and Emission Models: Could Plausible Errors Result in Air Quality Nonconformity? Preprint for the Transportation Research Board's 80 ${ }^{\text {th }}$ Annual Meeting, Washington D.C., Jan 2001.

Sheffi, Y. Urban Transportation Networks: Equilibrium Analysis with Mathematical Programing Methods. Engelwood Cliffs, New Jersey, 1984. 
Sheffi, Y. and W. Powell. An Algorithm for the Equilibrium Assignment Problem with Random Link Times. Networks 12, 1982, pp. 191-207.

Stopher, P.R. and A. H. Meyburg Urban Transportation Modeling and Planning. D.C. Health and Company, 1975.

Thompson, D., M. Baker, and D. Wade. Conformity: Long Term Prognosis for Selected Ozone Non-attainment Areas in California, January 1997, Preprint for the Transportation Research Board's $76^{\text {th }}$ Annual Meeting, Washington D.C.

UrbanSim Application Guide. University of Washington, Urban Simulation Project, 2000. (Accessed on 10/15/2000 at 16:00 GMT, at the UrbanSim Website: http://www.urbansim.org/Papers/Urbansim_Reference_Guide-09.pdf)

UrbanSim Reference Guide. University of Washington, Urban Simulation Project, 2000. (Accessed on 10/15/2000 at 16:00 GMT, at the UrbanSim Website: http://www.urbansim.org/Papers/Urbansim_Reference_Guide-09.pdf)

Waddell. P., A. Borning, M. Noth, N. Freier, M. Becke, and G. Ulfarsson. UrbanSim: A simulation System for Land Use and Transportation, 2001. (Accessed on 05/15/2001 at 16:00 GMT at the UrbanSim Website: http://www.urbansim.org/Papers/UrbanSim_NSE_Paper.pdf)

Zhao, Y., and K. M. Kockelman. The Propagation of Uncertainty Through Travel Demand Models. Presented at the Transportation Research Board's $80^{\text {th }}$ Annual Meeting, Washington D.C., Jan 2001.

\section{LIST OF TABLES}

Table 1: $\quad$ Sensitivity of Land Use Model Outputs to Inputs ........................... 16

Table 2: $\quad$ Sensitivity of Travel Model Outputs to Inputs............................. 17

Table 3: $\quad$ Comparison of Model Outputs with Observed Patterns..............18

\section{LIST OF FIGURES}

Figure 1: Partitioning the Input Probability Distribution............................. 19

Figure 2: The Evolution of Uncertainty in Model Outputs ........................... 20 


\section{TABLE 1 Sensitivity of Land Use Model Outputs to Inputs}

\begin{tabular}{|c|c|c|c|c|c|c|}
\hline \multirow[t]{2}{*}{ Variable } & \multicolumn{2}{|c|}{ Land Prices } & \multicolumn{2}{|c|}{ Occupancy Density } & \multicolumn{2}{|c|}{ Occupancy Rates } \\
\hline & Std. Coeff. & p-value & Std. Coeff. & p-value & Std. Coeff. & p-value \\
\hline \multicolumn{7}{|l|}{ Sensitivity Analysis for 1985} \\
\hline Constant & - & 0.000 & - & 0.000 & - & 0.000 \\
\hline Growth Rates & -0.732 & 0.000 & 0.986 & 0.000 & 0.997 & 0.000 \\
\hline Mobility Rates & -0.090 & 0.000 & 0.116 & 0.000 & 0.023 & 0.009 \\
\hline $\begin{array}{l}\text { Accessibility for Location } \\
\text { Choice Models }\end{array}$ & -0.004 & 0.515 & 0.002 & 0.905 & 0.013 & 0.152 \\
\hline $\begin{array}{l}\text { Accessibility for Land Price } \\
\text { Model }\end{array}$ & 0.673 & 0.000 & -0.010 & 0.467 & -0.005 & 0.552 \\
\hline Adjusted R-Squared & 0.997 & & 0.986 & & 0.994 & \\
\hline \multicolumn{7}{|l|}{ Sensitivity Analysis for 1990} \\
\hline Constant & - & 0.000 & - & 0.000 & - & 0.000 \\
\hline Growth Rates & -0.882 & 0.000 & 0.994 & 0.000 & 0.886 & 0.000 \\
\hline Mobility Rates & -0.046 & 0.001 & 0.041 & 0.001 & 0.004 & 0.951 \\
\hline $\begin{array}{l}\text { Accessibility for } \\
\text { Location Choice Models }\end{array}$ & -0.009 & 0.485 & 0.001 & 0.961 & 0.005 & 0.931 \\
\hline $\begin{array}{l}\text { Accessibility for Land } \\
\text { Price Model }\end{array}$ & 0.454 & 0.000 & -0.014 & 0.253 & -0.003 & 0.964 \\
\hline Adjusted R-Squared & 0.985 & & 0.989 & & 0.736 & \\
\hline \multicolumn{7}{|c|}{ Sensitivity Analysis for 1995} \\
\hline Constant & - & 0.000 & - & 0.000 & - & 0.000 \\
\hline Growth Rates & -0.705 & 0.000 & 0.865 & 0.000 & 0.862 & 0.000 \\
\hline Mobility Rates & -0.016 & 0.749 & 0.004 & 0.940 & 0.040 & 0.487 \\
\hline $\begin{array}{l}\text { Accessibility for Location } \\
\text { Choice Models }\end{array}$ & -0.004 & 0.930 & -0.003 & 0.963 & -0.015 & 0.798 \\
\hline $\begin{array}{l}\text { Accessibility for Land Price } \\
\text { Model }\end{array}$ & 0.552 & 0.000 & -0.004 & 0.934 & -0.006 & 0.920 \\
\hline Adjusted R-Squared & 0.792 & & 0.736 & & 0.732 & \\
\hline
\end{tabular}


TABLE 2 Sensitivity of Travel Model Outputs to Inputs.

\begin{tabular}{|c|c|c|c|c|c|c|}
\hline \multirow[t]{2}{*}{$\overline{\text { Variable }}$} & \multicolumn{2}{|c|}{ VHT } & \multicolumn{2}{|c|}{ VMT } & \multicolumn{2}{|c|}{ A verage Flow } \\
\hline & Std. Coeff. & p-value & Std. Coeff. & $p$-value & Std. Coeff. & p-value \\
\hline \multicolumn{7}{|l|}{ Sensitivity Analysis for 1985} \\
\hline Constant & - & 0.000 & - & 0.000 & - & 0.000 \\
\hline Growth Rates & 0.994 & 0.000 & 0.995 & 0.000 & 0.997 & 0.000 \\
\hline Mobility Rates & -0.012 & 0.043 & -0.003 & 0.612 & -0.042 & 0.000 \\
\hline $\begin{array}{l}\text { Accessibility for Location } \\
\text { Choice Models }\end{array}$ & 0.092 & 0.000 & 0.088 & 0.000 & 0.009 & 0.210 \\
\hline $\begin{array}{l}\text { Accessibility for Land Price } \\
\text { Model }\end{array}$ & 0.001 & 0.898 & 0.001 & 0.910 & -0.006 & 0.450 \\
\hline Adjusted R-Squared & \multicolumn{2}{|c|}{0.997} & \multicolumn{2}{|c|}{0.998} & \multicolumn{2}{|c|}{0.966} \\
\hline \multicolumn{7}{|l|}{ Sensitivity Analysis for 1990} \\
\hline Constant & - & 0.000 & - & 0.000 & - & 0.000 \\
\hline Growth Rates & 0.830 & 0.000 & 0.853 & 0.000 & 0.963 & 0.000 \\
\hline Mobility Rates & 0.001 & 0.987 & -0.002 & 0.976 & -0.041 & 0.188 \\
\hline $\begin{array}{l}\text { Accessibility for Location } \\
\text { Choice Models }\end{array}$ & 0.020 & 0.758 & 0.025 & 0.672 & 0.015 & 0.622 \\
\hline $\begin{array}{l}\text { Accessibility for Land Price } \\
\text { Model }\end{array}$ & 0.016 & 0.801 & 0.016 & 0.786 & 0.010 & 0.750 \\
\hline Adjusted R-Squared & \multicolumn{2}{|c|}{0.673} & \multicolumn{2}{|c|}{0.713} & \multicolumn{2}{|c|}{0.925} \\
\hline \multicolumn{7}{|l|}{ Sensitivity Analysis for 1995} \\
\hline Constant & - & 0.000 & - & 0.000 & - & 0.000 \\
\hline Growth Rates & 0.860 & 0.000 & 0.866 & 0.000 & 0.875 & 0.000 \\
\hline Mobility Rates & 0.050 & 0.394 & 0.041 & 0.473 & -0.001 & 0.980 \\
\hline $\begin{array}{l}\text { Accessibility for Location } \\
\text { Choice Models }\end{array}$ & 0.051 & 0.382 & 0.042 & 0.466 & 0.012 & 0.834 \\
\hline $\begin{array}{l}\text { Accessibility for Land Price } \\
\text { Model }\end{array}$ & 0.000 & 1.000 & 0.003 & 0.952 & 0.005 & 0.923 \\
\hline Adjusted R-Squared & \multicolumn{2}{|c|}{0.731} & \multicolumn{2}{|c|}{0.740} & \multicolumn{2}{|c|}{0.754} \\
\hline
\end{tabular}


TABLE 3 Comparison of Model Outputs with Observed Patterns.

\begin{tabular}{|cccccc|}
\hline Variable & \multicolumn{3}{c}{ Predicted } & Observed & \multirow{2}{*}{$\begin{array}{c}\text { Bias } \\
(\%)\end{array}$} \\
\cline { 2 - 4 } & High & Low & Average & & +12.2 \\
\hline Land Prices & 37.88 & 30.35 & 33.30 & 29.68 & +5.0 \\
\hline Occupancy Rates & 1.000 & 0.985 & 0.996 & 0.949 & +60.3 \\
\hline Occupancy Densities & 0.859 & 0.979 & 0.941 & 0.587 & -8.5 \\
\hline VHT & 3452 & 3380 & 3425 & 3744 & -8.9 \\
\hline VMT & 179551 & 175304 & 177951 & 195283 & -4.5 \\
\hline Average Flow & 6201 & 5987 & 6123 & 6408 & \\
\hline
\end{tabular}




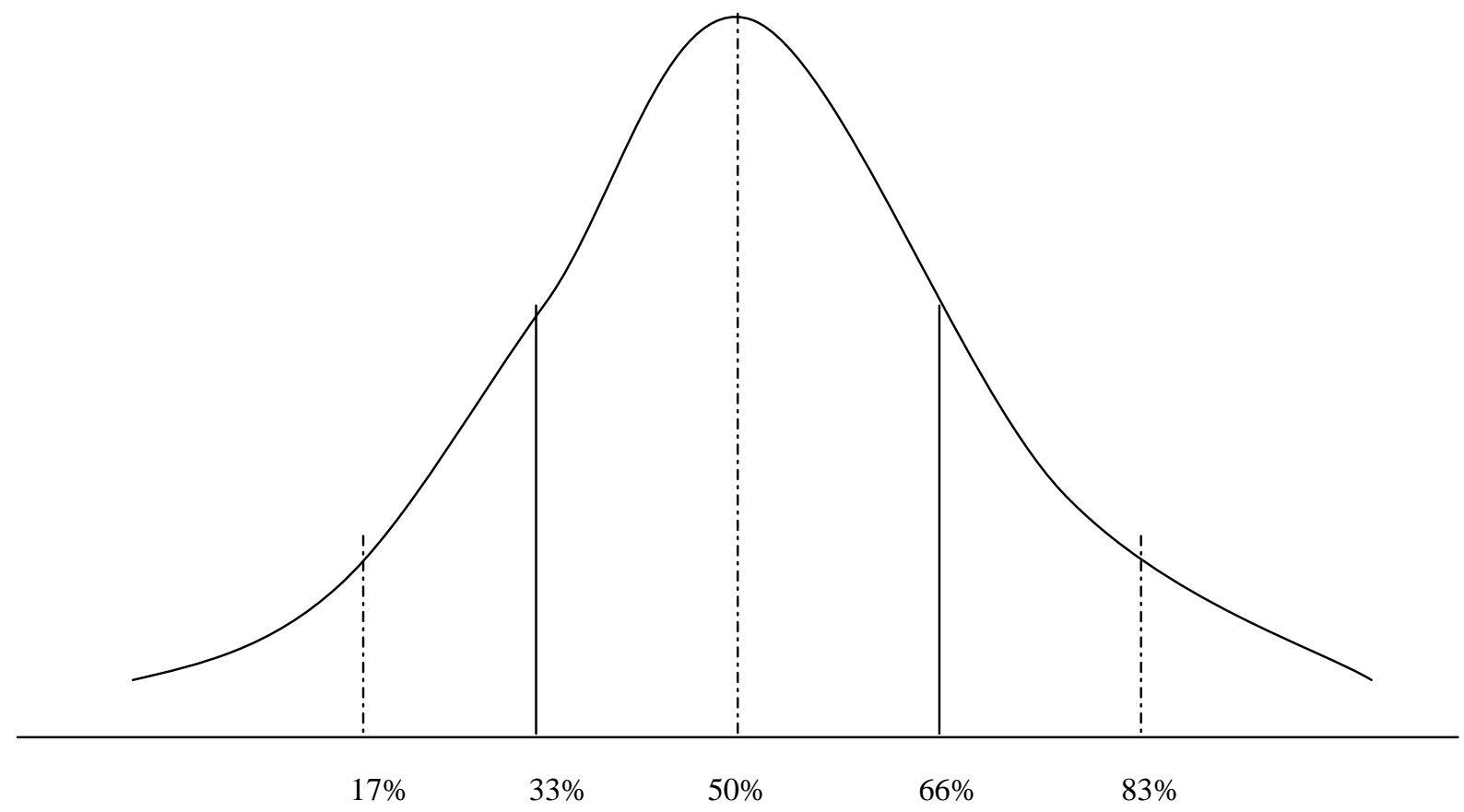

FIGURE 1 Partitioning the Input Probability Distribution. 


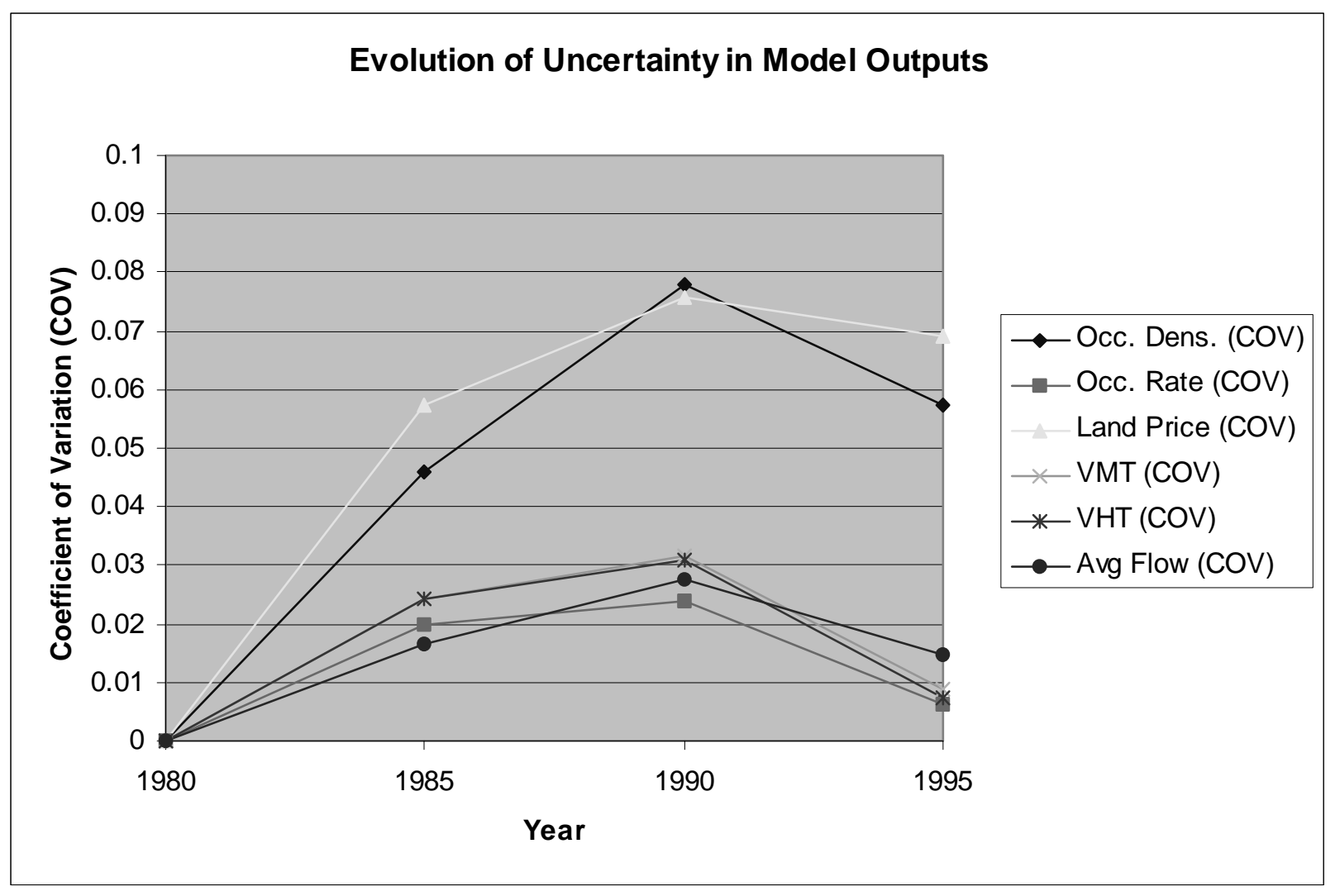

FIGURE 2 Evolution of Uncertainty in Model Outputs. 